\title{
Hepatectomia Direita Ampliada com Ressecção Parcial da Veia Cava para Metástase Colorretal: Relato de Caso
}

\author{
Extended Right Hepatectomy with Partial Resection Of The Vena Cava from \\ Colorectal Metastases: Case Report
}

\author{
SERGIO RENATO PAIS COSTA ${ }^{1}$; OLIIMPIAALVES TEIXEIRALIMA²; TÚLIO MARCOS RODRIGUES DACUNHA ${ }^{3}$; \\ ALOISIOFERNANDES SOARES ${ }^{4}$
}

\begin{abstract}
1. Especialista em Cancerologia Cirúrgica e Cirurgia do Aparelho Digestivo - Doutorando e Mestre em Cirurgia pela UNIFESP - Medico Colaborador do Serviço de Cirurgia Geral do Hospital Regional da Asa Norte - Brasília - Distrito Federal; ${ }^{2}$ Especialista em Cirurgia Geral - Mestre em Cirurgia pela UFPR - Médica Assistente do Serviço de Cirurgia Geral do Hospital Regional da Asa Norte - Brasília - Distrito Federal; ${ }^{3 .}$ Especialista em Cirurgia do Aparelho Digestivo - Chefe do Serviço de Cirurgia Geral do Hospital Regional da Asa Norte - Distrito Federal; ${ }^{4}$ Especialista em Cirurgia Geral - Doutor e Mestre em Cirurgia pela USP-RP - Chefe do Serviço de Cirurgia Geral do Hospital Regional da Asa Norte - Brasília - Distrito Federal.
\end{abstract}

\begin{abstract}
COSTA SRP; LIMA OAT; CUNHA TMR; SOARES AF. Hepatectomia Direita Ampliada com Ressecção Parcial da Veia Cava para Metástase Colorretal: Relato de Caso. Rev bras Coloproct, 2010;30(2): 232-235.

RESUMO: Introdução: A hepatectomia tem sido o tratamento padrão para metástase de origem colorretal (CR). Metástase com invasão da veia cava inferior (VCI) pode requerer ressecção combinada do fígado e VCI. Esta abordagem pode apresentar alto risco cirúrgico. Sangramento profuso e embolia gasosa são complicações intra-operatorias letais. Relato de Caso: Os autores relatam um caso de metástase colorretal única tratada com hepatectomia direita ampliada e ressecção parcial da veia cava com reconstrução primaria. Paciente apresentou boa evolução pós-operatória sem recidiva (um ano de seguimento). Conclusão: Ressecção da VCI e reconstrução combinada com hepatectomia pode ser satisfatoriamente realizada em casos selecionados. A falta de tratamentos alternativos e o prognostico reservado nos casos não operados justificam esta conduta agressiva. No entanto, esta abordagem deve ser realizada por equipe especializada em cirurgia hepática.
\end{abstract}

Descritores: Neoplasias colorretais; Hepatectomia; Metástase neoplásica; Neoplasias hepáticas/cirurgia, Neoplasias hepáticas/secundário; Taxa de Sobrevida.

\section{INTRODUÇÃO}

A hepatectomia tem sido considerada o tratamento curativo de escolha para as metástases de etiologia colorretal. Em revisão sistemática recentemente publicada, a taxa de sobrevida em cinco anos em 16 series incluindo somente ressecções R0 variou de 15 a $67 \%$ com mediana de $30 \%$. Paralelamente, com o maior conhecimento anatômico e da técnica operatória aliada a crescente melhoria nas condições de suporte tanto no intra quanto no pós- operatório tem levado a uma diminuição substancial da mortalidade dessa operação. Neste mesmo estudo, a mortalidade pós-operatória variou de 0 a $6,6 \%$ com mediana de $3 \%$ (1).

Diversos fatores prognósticos têm sido estudados em relação às metástases $\mathrm{CR}$. $\mathrm{O}$ número e tamanho das metástases assim como o intervalo de livre de doença entre o tumor primário e a lesão metastática, a taxa sérica do antígeno carcinoembrionario bem como as margens cirúrgicas tem sido apontadas como importantes fatores de sobrevida em longo prazo. Devido

Trabalho realizado no Hospital Regional da Asa Norte - Brasília - Distrito Federal - Brasil. 
aos avanços na cirurgia hepática tem havido uma maior liberdade nas indicações das hepatectomias para o tratamento das metástases, mormente nas de etiologia colorretal (1-7). Embora no passado a invasão vascular tenha sido considerada critério absoluto de irressecabilidade, com a propagação das técnicas de transplante e maior experiência em hepatectomias tecnicamente mais complexas, atualmente a invasão de vasos vitais como a veia cava não tem sido obstáculo para a ressecção de metástases CR em casos ultraselecionados $(8,9)$.

Os autores descrevem um caso de metástase única de etiologia $\mathrm{CR}$ com invasão da veia cava inferior submetida à hepatectomia direita ampliada e reconstrução vascular com boa evolução pós-operatória e sobrevida livre de doença de um ano.

\section{RELATO DE CASO}

Paciente de 50 anos, sexo feminino, submetida à colectomia total e ileostomia por tumor sincrônico de cólon (lesões em cólon sigmóide e transverso estádio T3N0M0), em janeiro de 2005 com reconstrução de trânsito após 11 meses. Não foi realizado tratamento adjuvante. Aos quatro anos de evolução, em janeiro de 2009, em seguimento de rotina foi observado a tomografia computadorizada do abdome metástase única de 7,5 cm em lobo direito do fígado (segmentos V-VIII) com invasão parcial da cava retro-hepática e presença de imagem de falha de enchimento compatível com trombo de $2 \mathrm{~cm}$. A doente apresentava excelente estado geral e não havia sinais de disseminação extra-hepática (clinica ou radiológica). A dosagem sérica de antígeno carcinoembrionario era de $81.2 \mathrm{ng} /$ $\mathrm{ml}$. Em virtude de a metástase ser única e a doente apresentar um longo intervalo livre de doença (entre tratamento do primário e aparecimento da metástase) foi proposta exploração cirúrgica. Foi submetida à laparotomia por incisão mediana, onde ao inventário encontrou-se grande tumor em lobo direito do fígado sem sinais de disseminação à distância, contudo com invasão parcial da veia cava inferior ate a entrada da veia renal direita que também se apresentava invadida. Diante da complexidade da ressecção foi realizada linfadenectomia do hilo hepático cujo exame de congelação intra-operatório mostrou-se negativo para neoplasia.

Dada a ressecabilidade da lesão com margem adequada, foi optado por prolongamento da incisão transversalmente a direita - incisão em J ou L invertido (figura 1), devido ao volume do tumor e sua intima relação com a veia cava e renal direita foi optado por exclusão vascular total do fígado.

Prosseguiu-se com dissecção completa das estruturas do hilo hepático seguida da dissecção e reparo da veia cava inferior supra e infra-hepática (Figuras 2 e 3). Por conseguinte foi realizado hepatectomia direita ampliada para segmento IV com secção do parênquima por digitoclasia e isquemia continua (com tempo total de 30 minutos) com ressecção parcial de veia cava, retirada de trombo tumoral (Figura 4) e ressecção da veia renal direita em monobloco com posterior nefrectomia direita mediante a incerteza de invasão do parênquima renal e da adrenal direitas. A reconstrução da veia cava foi realizada com síntese primária (anastomose cavo-caval). O histopatológico revelou adenocarcinoma colorretal metastático com invasão de veia cava, veia renal direita, glândula adrenal direita e ausência de linfonodos comprometidos (23 dissecados). No pós-operatório evoluiu com hipoproteinemia, anasarca e anemia que foram revertidas clinicamente. A doente foi submetida a duas

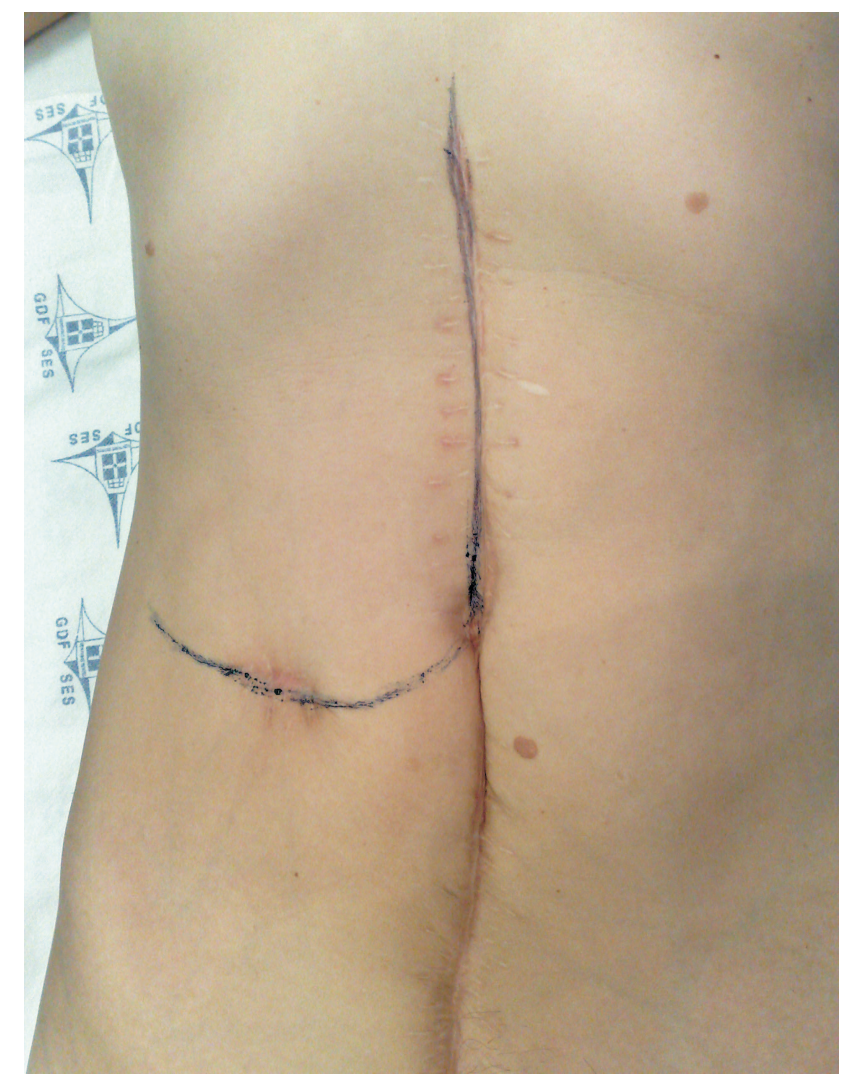

Figura 1 - Incisão em J ou L invertido. 


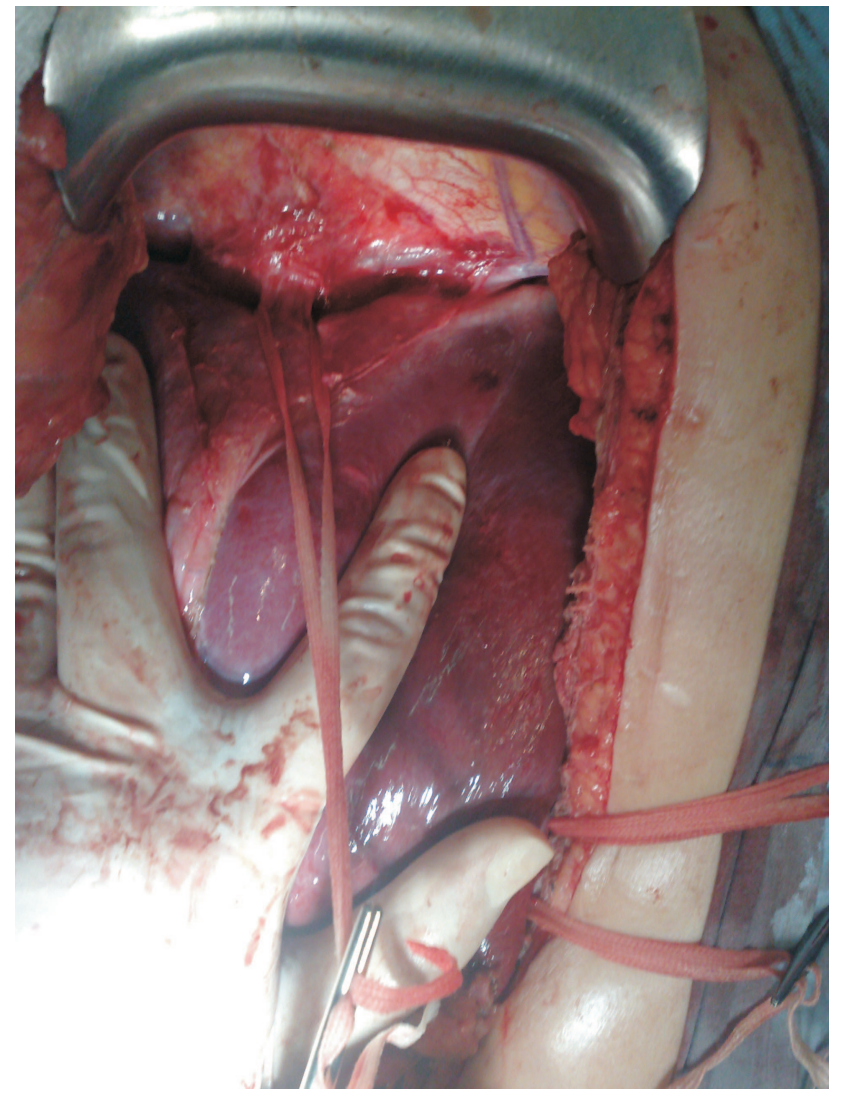

Figura 2 - Isolamento e Reparo da Veia Cava (Supra + InfraHepatica).

laparotomias no período, por síndrome compartimental abdominal com ressutura da parede abdominal. Evoluiu com hérnia incisional que foi corrigida aos seis meses da hepatectomia. Com um ano de seguimento se apresentava assintomática e sem recidiva da doença.

\section{DISCUSSÃO}

A ressecção de metástases hepáticas de etiologia CR tem sido considerada o tratamento curativo padrão. Com melhor conhecimento da biologia tumoral, diversos estudos prognósticos tem apontado que alguns fatores preditores de sobrevida em longo prazo são de suma importância na escolha pelo tratamento cirúrgico. Fong e cols, em 1999 publicaram uma serie de 1001 doentes com metastases CR ressecadas no Memorial Sloan-Kettering Cancer Center nos Estados Unidos da América. A partir de uma analise uni e multivariada observaram cinco critérios prognósticos de importância para sobrevida em longo prazo: 1) comprometimento linfonodal do tumor primário; 2) intervalo livre de doença do diagnóstico da

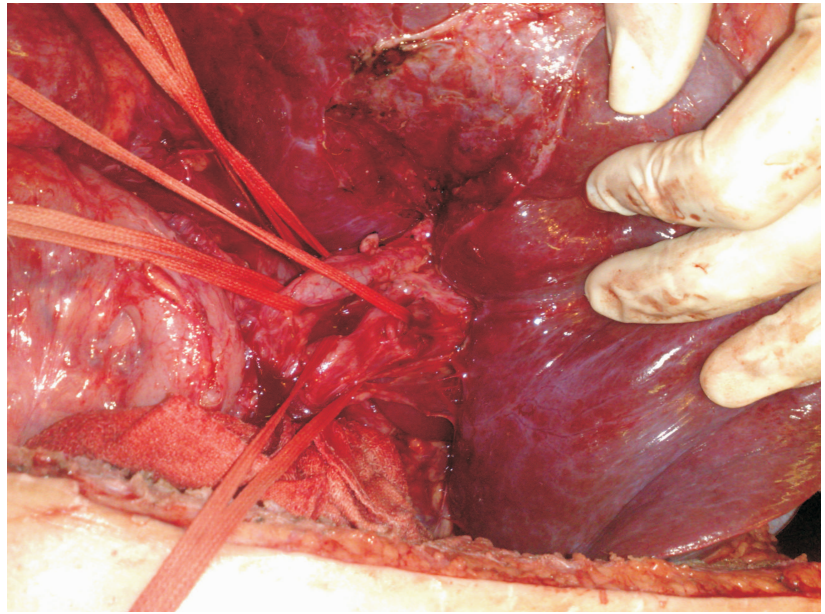

Figura 3 - Isolamento e Reparo das estruturas do hilo hepático.

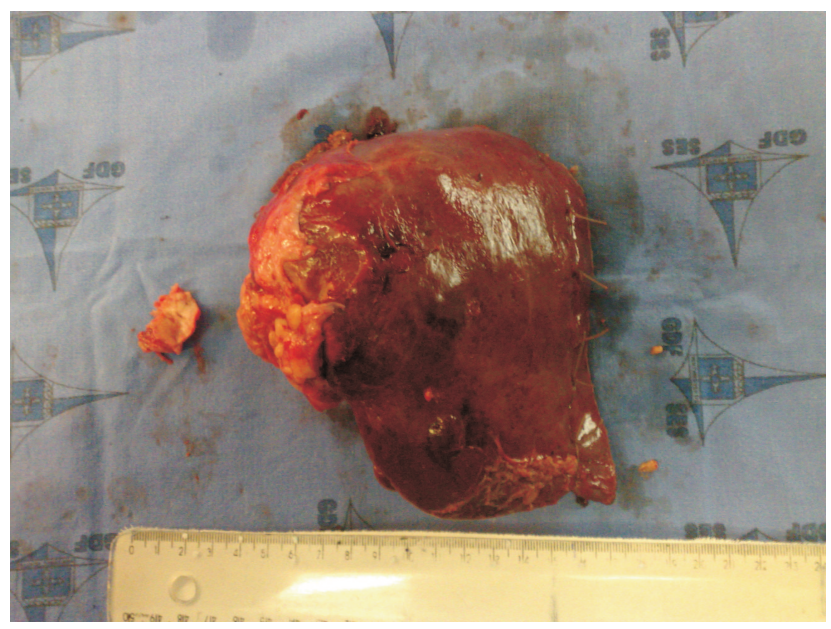

Figura 4-Produto de Hepatectomia Direita + ressecção parcial da veia cava retro-hepática.

lesão primaria e descoberta da metástase maior que 12 meses; 3)número de metástases maior que uma; 4) Tamanho da maior metástase maior que cinco centímetros; 5) nível serico de antígeno carcinoembrionario maior que $200 \mathrm{ng} / \mathrm{dl}$. Em estudo previamente publicado em nosso meio pelo presente autor, metástase única assim como ausência de linfonodos comprometidos tanto no tumor primário quanto no hilo hepático também foram os principais fatores prognosticos de sobrevida em longo prazo a analise uni e multivariada. Paralelamente, na opinião dos autores, a linfadenectomia hilar durante a hepatectomia e de fundamental importância para melhor conhecimento do prognostico do doente e seleção para terapia adjuvante (5). Em que pese à alta probabilidade de morbidade e mortalidade em virtude da invasão de VCI, os autores optaram por essa abordagem agressiva haja vista que baseado nos critérios 
Hepatectomia Direita Ampliada com Resseccão Parcial da

Veia Cava para Metástase Colorretal: Relato de Caso Sergio Renato Pais Costa e Cols.
Vol. 30 prognósticos apenas o tamanho da metástase foi critério preditivo negativo.

Mesmo assim de acordo com o escore citado por Fong e cols, a chance da presente doente sobreviver cinco anos com somente um critério prognóstico negativo é de cerca de $50 \%$. Desta maneira uma ressecção ultra-radical em uma doente jovem com expectativa de sobrevida longa pode estar justificada (17).

Paralelamente, a falta de abordagens terapêuticas alternativas com resultados similares a ressecção associado ainda ao melhor conhecimento da cirurgia hepática tem impulsionado sobremaneira a ampliação das indicações das hepatectomias. A expectativa do doente submetido à quimioterapia exclusiva raramente suplanta cinco anos. Em relação ao tratamento cirúrgico das metástases CR, abordagens mais agressivas tem sido mais frequentemente admitidas como hepatectomias extremas, em dois tempos (com ou sem embolização portal) associados à ablação por radiofrequiência ou quimioterapia neo-adjuvante (1-7). O melhor conhecimento das técnicas de transplante também tem ampliado o arsenal de condutas terapêuticas mais agressivas como hepatectomias com de exclusão vascular hepática total, bypass veno-venoso e perfusão hipotérmica (in situ, ante situm ou ex situ). Estas condutas assaz invasivas têm sido baseadas na maior expectativa de vida do doente operado frente ao tratado com terapia sistêmica exclusiva encontrada na literatura, quando uma ressecção R0 e realizada em um doente com metástase hepática $\mathrm{CR}$ única sua chance de sobrevida em cinco anos pode chegar ate $55 \%(8,9)$.

Embora no passado a invasão de estruturas vasculares vitais como a VCI tenha sido considerada critério de irressecabilidade, mais recentemente algumas series tem demonstrado a factibilidade de uma ressecção vascular com reconstrução em casos ultraselecionados $(8,9)$.
Esta abordagem tem sido mais frequentemente realizada principalmente em virtude da popularização de técnicas mais refinadas oriundas do transplante hepático. O tipo de reconstrução mais freqüente tem sido a síntese primária como no presente caso, mas também reconstruções com pericárdio bovino ou próteses vasculares (Gorotex ou PTFE) podem ser utilizadas, mormente quando os defeitos são mais extensos. No entanto, mesmo em centros de referencia a mortalidade desse tipo de ressecção tem variado de 4,5 a $9 \%$ e morbidade igualmente elevada de $64 \%$. Hemming e cols (8) descreveram uma serie de 22 pacientes com tumor hepático maligno com invasão da VCI e hepatectomia associada a ressecção da VCI. A sobrevida global desta serie em cinco anos foi de 33 $\%$ com mediana de seguimento de 26 meses. Desta casuística, vinte doentes seguiram ressecção com exclusão vascular do fígado sendo que em seis casos foi realizada reconstrução vascular primária. Azoulay e cols (9) também em casuística de 22 pacientes com tumores hepáticos malignos ressecados que envolviam a VCI observaram uma sobrevida em cinco anos de $38 \%$, com uma mediana de seguimento de 19 meses cerca de 11 doentes estavam vivos sendo cinco sem recidiva tumoral. . Doze doentes seguiram exclusão vascular total do fígado e reconstrução primaria da VCI como no caso descrito. Estes autores concluíram que em virtude da falta de tratamentos alternativos e do prognostico ruim dos casos não ressecados esta abordagem agressiva estaria justificada.

\section{CONCLUSÃO}

A ressecção de metástase hepática com invasão da veia cava inferior e reconstrução primaria e factível de ser realizada à custa de uma alta morbidade podendo oferecer um prolongamento de sobrevida em casos ultra-selecionados (metástase única, ausência de metástase linfonodal e intervalo livre de doença longo).

\footnotetext{
ABSTRACT: Introduction: Hepatectomy has been standard treatment for metastases from colorectal origin (CR). Metastases with inferior vena cava (IVC) involvement may require combined resection of the liver and IVC. This approach may present high surgical risk, Both profuse bleeding and gas embolism are letal intraoperative complications. Case: The authors present a case of single hepatic CR metastasis that was treated by means extended right hepatectomy with partial inferior vena cava resection and primary reconstruction. Patient present good postoperative course without neoplasm recurrence (one year follow-up period). Conclusion: Resection of VCI and combined reconstruction with hepatectomy may be satisfactorily done in selected cases. Lack of alternative treatments associated poor prognosis of untreated cases has justified this aggressive conduct. Therefore, this approach should be realized by hepatic surgery expertise team.
}

Key words: Colorectal neoplasm, Hepatectomy; Neoplasm metastasis; Liver neoplasm/surgery; Liver neoplasm/secondary; Survival rate. 


\section{REFERÊNCIAS}

1. Simmonds PC, Primrose JN, Colquitt JL, Garden OJ, Poston GJ, Rees M. Surgical resection of metastases from colorectal cancer: A systematic review of published studies. British Journal of Cancer 2006; 94: 982 - 99.

2. Fong Y, Fortner J, Sun RL, Brennan MF, Blumgart LH. Clinical score for predicting recurrence after hepatic resection for metastatic colorectal cancer : analysis of 1001 consecutive cases. Ann Surg 1999; 230 : 309-21.

3. Adam R. Tratamento das Metastases Hepaticas do Câncer Colorretal. In : Correia MM, Mello EIR, Santos CER. Eds Cirurgia do Câncer Hepatobiliar. Primeira edicao, Rio de Janeiro. Revinter ; 2003 : p. 139-46.

4. Choi EA, Rodgers SE, Ahmad SA, Abdalla EK. In : Feig BW, Berger DH, Fuhrman GM Eds. The M.D. Anderson Surgical Oncology Handbook. 4 Th Edition, Philadelphia : Lippincott \& Wilkins ; 2006. p. 238-47.

5. Minagawa M, Makuuchi M, Torzilli G, Takayama T, Kawasaki $\mathrm{S}$, Kosuge $\mathrm{T}$ et al. Extension of the Frontiers of Surical Indications in the Treatment of Liver Metastases from Colorectal Cancer. Ann Surg 2000 ; 231 (4) : 487-99.
6. Scheele J, Altendorf-Hofmann A. Surgical Treatment of Liver Metastases. In: Blumgart LH \& Fong Y Eds. Surgery of the Liver and Biliary Tract. 3 th Edition. Edinburgh WB Saunders 2000: p. 1475-1502.

7. Costa SRP, Horta SHC, Henriques AC, Waisberg J, Speranzini MB. Hepatectomia para o Tratamento de metástases colorretais e não-colorretais: Análise Comparativa em 30 casos operados. Rev bras Coloproctol 2009; 29 (2): 216-25.

8. Hemming AW, Reed AI, Langham MR, Fujita S, Howard JR. Combined Resection of the Liver and Inferior Vena Cava for Hepatic Malignancy. Ann Surg 2004; 239 (5): 712-21.

9. Azoulay D, Andreani P, Maggi U, Salloum C, Perdigao F, Sebagh M, et al. Combined Liver Resection and Reconstruction of the Supra-Renal Vena Cava. The Paul Brousse Experience. Ann Surg 2006; 244 (1): 80-88.

\section{Endereço para correspondência:} SERGIO RENATO PAIS COSTA

Avenida W3

Brasilia - DF - Brasil

CEP: $14400-200$

E-mail: srenatopaiscosta@ hotmail.com 